\title{
Proximal Junctional Kyphosis in Adult Spinal Deformity: Definition, Classification, Risk Factors, and Prevention Strategies
}

\author{
Hong Jin Kim ${ }^{1,}$, Jae Hyuk Yang ${ }^{2,}$, Dong-Gune Chang ${ }^{1}$, Se-Il Suk ${ }^{1}$, Seung Woo Suh ${ }^{2}$, \\ Sang-Il Kim ${ }^{3}$, Kwang-Sup Song ${ }^{4}$, Jong-Beom Park ${ }^{3}$, Woojin Cho ${ }^{5}$ \\ ${ }^{1}$ Department of Orthopaedic Surgery, Inje University Sanggye Paik Hospital, Inje University College of Medicine, Seoul, Korea \\ ${ }^{2}$ Department of Orthopaedic Surgery, Korea University Guro Hospital, Korea University College of Medicine, Seoul, Korea \\ ${ }^{3}$ Department of Orthopaedic Surgery, College of Medicine, The Catholic University of Korea, Seoul, Korea \\ ${ }^{4}$ Department of Orthopaedic Surgery, Chung-Ang University Hospital, Chung-Ang University College of Medicine, Seoul, Korea \\ ${ }^{5}$ Department of Orthopaedic Surgery, Montefiore Medical Center, Albert Einstein College of Medicine, Bronx, NY, USA
}

Proximal junctional problems are among the potential complications of surgery for adult spinal deformity (ASD) and are associated with higher morbidity and increased rates of revision surgery. The diverse manifestations of proximal junctional problems range from proximal junctional kyphosis (PJK) to proximal junctional failure (PJF). Although there is no universally accepted definition for PJK, the most common is a proximal junctional angle greater than $10^{\circ}$ that is at least $10^{\circ}$ greater than the preoperative measurement. PJF represents a progression from PJK and is characterized by pain, gait disturbances, and neurological deficits. The risk factors for PJK can be classified according to patient-related, radiological, and surgical factors. Based on an understanding of the modifiable factors that contribute to reducing the risk of PJK, prevention strategies are critical for patients with ASD.

Keywords: Proximal junctional problems; Proximal junctional kyphosis; Proximal junctional failure; Adult spinal deformity; Prevention strategies

\section{Introduction}

Adult spinal deformity (ASD) is a heterogeneous spectrum of abnormalities of the thoracic or thoracolumbar spine that significantly affects health-related quality of life (HRQoL) [1-3]. The incidence of ASD has increased in concert with longer life expectancies and the expanding population of healthy older individuals. ASD surgery is closely associated with complications, and revision sur- gery is common because multilevel fusions are frequently performed to address deformities $[1,2,4]$. The development of kyphosis at the transition between fused and mobile motion segments in the ASD area has emerged as one of the most important complications $[1,4,5]$.

Proximal junctional problems after ASD surgery range from proximal junctional kyphosis (PJK) to proximal junctional failure (PJF) [4]. PJK is considered a radiological phenomenon, reflecting pathological changes that

Received Nov 10, 2020; Revised Feb 9, 2021; Accepted Feb 15, 2021

Corresponding author: Dong-Gune Chang

Department of Orthopaedic Surgery, Inje University Sanggye Paik Hospital, Inje University College of Medicine, 1342 Dongil-ro, Nowon-gu, Seoul 01757, Korea

Tel: +82-2-950-1284, Fax:+82-2-950-1287, E-mail: dgchangmd@gmail.com

*These two authors contributed equally to this work as a co-first author. 
develop around the adjacent segment after long instrumented posterior fusion [6]. PJK has a varying spectrum of presentation, from no clinical symptoms to the need for revision surgery [6,7]. Although there are no universally accepted definitions for PJK, PJK is commonly defined as a proximal junctional sagittal Cobb angle $\geq 10^{\circ}$ that is at least $10^{\circ}$ greater than the preoperative measurement $[4,5]$. $\mathrm{PJF}$ is a progression of PJK associated with features of structural failure, such as vertebral body fracture, posterior ligament complex (PLC) development, and vertebral subluxation [7]. PJF is associated with higher levels of pain, gait disturbances, and neurological deficits warranting revision surgery $[4,6,7]$.

The risk factors for PJK are not fully understood; however, PJK is commonly categorized according to patientrelated, radiological, and surgical factors [4,5]. Preventing PJK is important because there are no well-established management guidelines known to minimize the risk of revision surgery [8]. In this article, we discuss the current concepts surrounding proximal junctional problems, including the definitions, classification, risk factors, and strategies for preventing PJK. This review aims to provide comprehensive background information on PJK, including prevention strategies.

\section{Definition of Proximal Junctional Kyphosis and Proximal Junctional Failure}

There is presently no consensus regarding the precise definition of PJK [7], which can vary in the literature. A number of authors have suggested that PJK represents a broad spectrum of diagnoses that range from asymptomatic radiographic findings to those requiring revision surgery $[4,9]$. By its terminology, PJK is characterized by abnormal kyphotic deformity that occurs at the uppermost instrumented vertebrae (UIV) after posterior spinal fusion [10]. Given the radiological differences of $10^{\circ}$ for scoliosis and $11^{\circ}$ for kyphosis, Glattes et al. [11] proposed the definition of PJK as a proximal junctional sagittal Cobb angle (between the lower endplate of the UIV and the upper endplate of the two supra-adjacent vertebrae) of $10^{\circ}$ or more that is at least $10^{\circ}$ greater than the preoperative measurement in patients with ASD (Fig. 1). However, this definition did not take into account any physiological basis, including the postoperative disruption of the soft tissue, the facet capsule, and the interspinous ligament above the instrumented level, as among the most important factors

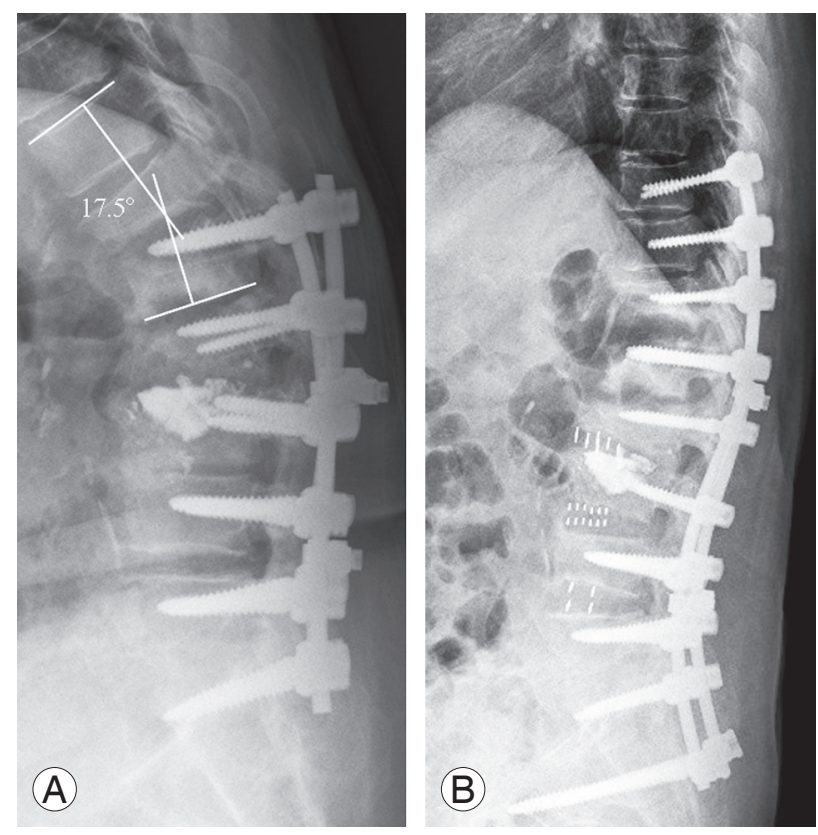

Fig. 1. Radiographs of a patient with proximal junctional kyphosis (PJK). Preoperative lateral radiograph of a 77-year-old woman with PJK showing proximal junction angle of $17.5^{\circ}$ (A). Postoperative 3-month follow-up lateral radiograph after revision surgery from $\mathrm{T} 10$ to the sacrum (B).

of PJK [4-6]. Reflecting these factors, Helgeson et al. [12] proposed another definition for $\mathrm{PJK}$, which specifies a proximal junctional angle (PJA) at least $15^{\circ}$ from the caudal endplate of the UIV to the cephalad endplate of one or two vertebrae above the UIV. After a large, multicenter retrospective study of patients with ASD, Hostin et al. [13] defined PJK as a Cobb angle formed by the lower endplate of the UIV and the upper endplate of two supra-adjacent vertebrae of $15^{\circ}$ or greater above the UIV. O'Shaughnessy et al. [14] and Bridwell et al. [15] used $20^{\circ}$ as their cutoff value, given that this was the angle associated with poorer patient-reported outcomes among patients who underwent primary adult scoliosis surgery. Although a cutoff value of $15^{\circ}$ or $20^{\circ}$ could reflect the physiological factors and clinical outcomes for PJK, these values are too narrow to be the standard for PJK revision surgery [16]. Although a cutoff value of $10^{\circ}$ could be applied in PJK, the final decision by spinal specialists should take into account the scale of the revision surgery, the radiological development, and the clinical outcomes.

In addition to the definition of PJK mentioned above, approximately 6-7 other definitions related to PJK have been described and used in previous studies, and there has been debate on the definition of PJK. According to a study by Ton et al. [9], none of the measurement methods 
for PJK presented until recently have shown an association between postoperative PJK and the patient's Oswestry Disability Index (ODI) score.

Considering that PJK is one of the nonsymptomatic complications that can occur after surgery in ASD and that checking for PJK after surgery can prevent additional problems that might occur in the future, it would be better to use the definition of PJK for progression of $10^{\circ}$ or more based on UIV+2 as defined by Glattes et al. [11]. This definition has higher sensitivity for PJK than other definitions and high reproducibility when measuring UIV+2 at either the upper thorax or thoracolumbar junction as well as considering the distribution pattern of external force that can cause fractures or PJK around UIV in biomechanical studies [17].

PJF is a progressive form of PJK characterized by features including vertebral fracture at the UIV or the $\mathrm{UIV}+1$ level, subluxation between the UIV and UIV +1 , failure of fixation, and neurological deficits, which could require revisional proximal extended fusion [7]. Yagi et al. [18] defined PJF as symptomatic PJK requiring any type of surgery. PJF is characterized by structural failure and mechanical instability, which can involve vertebral body fracture, implant pullout or breakage, and disruption of the posterior ligament complex [16]. Based on these studies, PJF can be defined as a form of PJK that requires surgical treatment due to structural failure and mechanical instability, which can be attributed to four broad classes of failure: fracture, spondylolisthesis, implant failure, and PJA progression [4]. Compared with PJK, PJF is associated with significantly poorer clinical symptoms and ODI scores [16].

\section{Classification of Proximal Junctional Kyphosis}

PJK is associated with a broad range of manifestations and presentations, from asymptomatic patients to those who require revision surgery $[4,5]$. To determine the need for revision surgery, there have been many attempts to better describe specific types of PJK [4]. In 2012, Yagi et al. [18] proposed a representative classification system that describes PJK by type (1=ligamentous failure; $2=$ bone failure; $3=$ implant or bone interface failure), grade (A, $\mathrm{B}$, or $\mathrm{C}$ corresponding to an increase in PJA of $10^{\circ}-19^{\circ}, 20^{\circ}-29^{\circ}$, or greater than $30^{\circ}$, respectively), and spondylolisthesis $(\mathrm{N}$, no obvious spondylolisthesis above the UIV; S, spondy- lolisthesis above the UIV). The authors also reported that most cases of PJF were type $2 \mathrm{~N}$ and that most cases with neurologic deficits were type $2 \mathrm{~S}[17,18]$. Although this scheme provides concise information about $\mathrm{PJK}$ for spinal specialists, it does not offer guidance for managing PJK [10].

Hart et al. [16] proposed the PJK Severity Scale, which has six components: neurological deficit, focal pain, instrumentation problems, change in kyphosis/PLC integrity, UIV/UIV+1 fracture, and UIV level. These components are assigned severity scores, which can be summed to derive a total score. If the total severity score is 7 or greater, revision surgery should be considered. A prospective study to determine the validity of the total scores is currently in progress $[10,19]$.

\section{Risk Factors for Proximal Junctional Kyphosis}

The pathogenesis of PJK is multifactorial, and its proposed mechanisms include (1) extensive paraspinal muscle dissection at the UIV; (2) disruption of the PLC, including the supraspinous and interspinous ligaments; (3) improper end-vertebra selection; (4) severe proximal disk degeneration; (5) compression fracture at the most instrumented vertebra; (6) instrumentation failure at the proximal construct; and (7) facet violation $[6,20]$. The risk factors for PJK can be divided into surgical, radiological, and patient-related factors [4].

The suggested patient-related risk factors include older age, high body mass index (BMI), and low bone mineral density (BMD, including osteopenia and osteoporosis) [4]. Older age ( $>55$ years) is among the major patientspecific risk factors commonly associated with revision surgery $[4,7]$. The degeneration and muscular atrophy associated with aging facilitate the development of PJK [19]. The association between age and PJK can be explained by age-dependent disk changes, facet joint degeneration, and atrophied paraspinal musculature [20]. Moreover, Pennington et al. [21] found that the size of the paraspinal muscle was associated with the ability to maintain sagittal correction, suggesting independent risk factor and suggested the PJK can be predicted more positive postoperative global sagittal alignment and smaller paraspinal musculature at the UIV in the view of patientrelated factor. Bridwell et al. [15] reported that BMI was significantly associated with the presence of comorbidities 
associated with the development of a PJA $>20^{\circ}$; however, a meta-analysis by Kim et al. [20] demonstrated that BMI was not associated with PJK or PJF. Bridwell et al. [15] also reported that low BMD increased the risk of PJK because proximal junctional problems result from fractures and subluxation at the UIV [4]. Patients with osteoporosis or osteopenia have been shown to have twice the odds of developing PJK, which is consistent with the relationship between low BMD and higher PJK incidence [20].

In terms of radiological risk factors, preoperative and postoperative sagittal malalignment among patients with ASD was reported to increase the risk of PJK, which is important for ASD treatment $[1,20]$. If compensatory mechanisms, such as pelvis rotation and extension of adjacent segments and legs, cannot compensate for sagittal malalignment preoperatively, patients tend to experience anterior sagittal imbalance and a tendency for anterior inclination $[1,22]$. Thus, older patients with ASD might have smaller preoperative lumbar lordosis (LL), larger preoperative pelvic tilt (PT), smaller LL-thoracic kyphosis (TK), larger pelvic incidence (PI)-LL, and larger preoperative sagittal vertical axis (SVA) and global spinal alignment $(\mathrm{TK}+\mathrm{LL}+\mathrm{PI})$. It is easy to understand why these preoperative radiological parameters are associated with PJK. The significant correlations between increasing age and these radiological parameters further verify our previous findings that age is a risk factor for the occurrence and development of PJK $[4,16,17]$. Higher TK, PJA, PI, and LL increase the risk of PJK [7]. Larger differences between preoperative TK and LL, lower sacral slope, and pelvic retroversion have also been associated with the development of PJK [4]. Local sagittal alignment parameters can also contribute to the development of PJK [4]. A preoperative PJA $>5^{\circ}$ has been reported to increase the risk of PJK and PJF $[4,7]$. In a meta-analysis evaluating risk factors for PJK development, Kim et al. [20] reported that a higher preoperative SVA is significantly associated with poorer radiographic sagittal misalignment, which can predispose patients to proximal junctional problems. Therefore, PJK is associated with a greater preoperative SVA as well as greater SVA correction.

For postoperative sagittal balance, a definition of ideal sagittal balance was needed. Various studies have been conducted for ideal sagittal balance in elderly patients, with Barrey et al. [23] and Roussouly et al. [24] suggesting a classification according to the type of compensation in consonance with the aging process in elderly patients.
With increased age, LL tends to decrease with pelvis rotation (increased PT) to maintain whole sagittal balance, which might result from lumbar degenerative diseases and low back pain [23]. Roussouly et al. [24] not only classified spinal shapes of healthy populations into five types but also proposed algorithms for restoring sagittal alignment by elucidating the correspondences between PI and spinal degenerative shape. Sebaaly et al. [25] reported ignoring the algorithm of Roussouly et al. [24] for ASD surgery because it increases the risk of mechanical complications three-fold. The combined loss of bony disk support by degeneration, osteoporosis, and muscle weakness promotes spinal imbalance in the elderly, inducing spinal kyphosis with or without compensation by pelvis retroversion, depending on the PI value.

Based on the results of these studies, other studies have been conducted to perfectly balance the sagittal plane when performing surgery. One study found that the degenerative changes in LL (especially lower lumbar) due to the aging process had a significant causal relationship with PI and that a mismatch between PI and LL was related to the ODI of patients before and after surgery [22]. Overcorrection for patients with ASD has been reported to increase the rate of PJK [4]. SVA overcorrection and increased postoperative LL was found to contribute to the development of PJK because they did not consider the age-specific sagittal alignment $[4,5]$. There are no definitive universal targets for spinopelvic measurements because patients with ASD have age-specific target parameters [26].

Proximal implant construction and modern pedicle screw stiffness have been recognized as the most important surgical risk factors for PJK [4]. Pedicle-only constructs have been associated with an increased PJK incidence compared with hook and hybrid constructs [12]. PLC disruption, including the posterior tension band and intervertebral elements, has been proposed as an irreversible risk factor for PJK development [7]. Similarly, PJK is 3 times more likely to develop in patients who have undergone a combined anterior-posterior approach than in patients who have undergone posterior-only fusion [27]. The choice of UIV also influences the development of PJK [11]. A UIV in the thoracolumbar spine can predispose patients to vertebral subluxation and fractures, which subsequently increase the risk of PJK and PJF [4]. A UIV at the upper thoracic level is associated with subluxation and soft tissue failure, but a UIV at the lower thoracic level is 


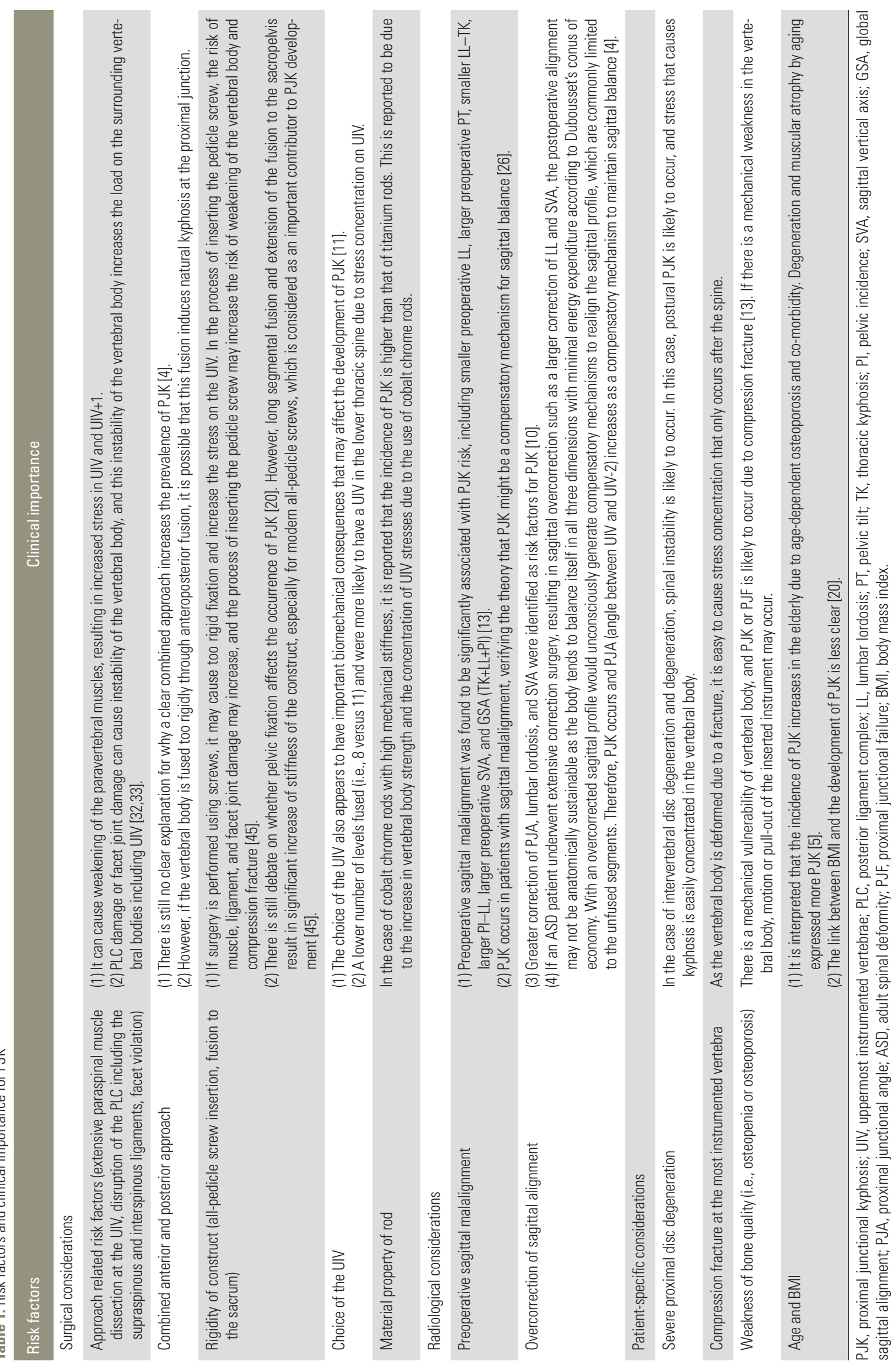


associated with secondary vertebral body fractures $[13,28]$. Bridwell et al. [15] reported that a UIV at the lower thoracic level was associated with a higher incidence of PJK than a UIV at the upper thoracic level. The risk factors for PJK and the clinical importance of interpreting risk factors are described in detail in Table 1.

\section{Methods for Preventing Proximal Junctional Kyphosis}

There are no well-established methods for preventing PJK. Based on etiologic and risk factors, Lau et al. [10] recommended the following modifiable factors and approaches for minimizing PJK risk: (1) extending fusion to include levels with baseline segmental kyphosis angles $>5^{\circ}$; (2) reducing instrumentation stiffness; (3) choosing composite metals; (4) using fewer implants, (5) more distal osteotomies; (6) less soft tissue destruction at the UIV; (7) attempting to achieve optimal spinal balance; and (8) using a transition rod.

\section{Patient-related considerations}

Although aging is irreversible, BMI and BMD can be modified by lifestyle changes and interventions by spine specialists $[4,5]$. For high BMI, lifestyle modifications promoting weight loss and nutrition counseling can help reduce the risk of PJK [4]. Osteopenia and osteoporosis are associated with vertebral fractures and pedicle screw loosening at the UIV [6]; osteoporosis treatment should therefore be considered for preventing PJK. The use of bisphosphonate remains controversial because of spinal fusion inhibition; however, teriparatide improves bone quality and reinforces fusion $[4,6,7]$. The use of teriparatide for 18 months after ASD surgery has been shown to significantly reduce PJF incidence, enhance fusion, and reduce pedicle screw loosening [4]. In addition, vertebral augmentation with cement can be performed. The addition of a cement mantle around a pedicle screw improves screw fixation and distributes stresses in the adjacent trabeculae, thereby reducing the tendency for screw loosening and pullout [29]. This process can be employed to treat osteopenia and osteoporosis and prevent PJK $[4,5]$. Surgeons can also choose the type of fixation, using either a large-diameter pedicle screw or a long pedicle screw (tricortical fixation through anterior cortical fixation of the vertebral body). Screw reinforcement with the addition of sublaminar wires or lamina hooks can increase the fixation strength by increasing the pullout strength, stiffness, and torsional stability in osteoporotic bone [30].

Recent studies have emphasized the importance of paraspinal muscle quality [31]. Kim et al. [20] studied the correlation between thoracolumbar muscularity (crosssectional area of muscle-vertebral body ratio $\times 100)$ and fatty degeneration (signal intensity of muscle-subcutaneous fat ratio $\times 100$ ) and PJK after surgery. The authors reported that patients with PJK had lower thoracolumbar muscularity and higher fatty degeneration than patients without PJK before surgery.

Pennington et al. [21] reported that preoperative paraspinal muscle size was strongly associated with PJK. However, there are few studies on how much paravertebral muscle atrophy and which muscle atrophy is related to PJK. Nevertheless, we recommend checking the quality of the paraspinal muscle using preoperative MRI scans. If atrophy or progressed fatty degeneration of the paravertebral muscle is confirmed, surgeons should pay close attention to prevent muscle and ligament damage when performing surgical treatment, and percutaneous screw insertion at the UIV is recommended to reduce muscle and ligament damage $[32,33]$.

\section{Radiological considerations}

Regarding mechanical failure after spinal surgery, the inadequate restoration of sagittal alignment has largely been attributed to the characteristics of load distribution [34]. The Scoliosis Research Society-Schwab classification of ASD first employed sagittal modifiers (PI/LL mismatch, PT, and SVA) to achieve satisfactory alignment and HRQoL $[22,35]$. Sagittal parameters including SVA, TK, LL, and PI should be included in PJK risk stratification considerations. Achieving optimal sagittal alignment is critical for preventing PJK [26]. Generally, correcting the PI/LL mismatch is a well-recognized tenet of ASD management [5]. Patients with a high PI $\left(>70^{\circ}\right)$ require slightly less LL, but those with a low PI $\left(<40^{\circ}\right)$ require slightly more LL. Schwab et al. [36] introduced the optimal target between LL and PI, which should be within $9^{\circ}$. With the association between spinopelvic variables and HRQoL, age-in relation to optimal postoperative spinopelvic measurements-is an important consideration for preventing PJK [1,4]. In terms of age-adjusted alignment goals, an SVA of $0 \mathrm{~cm}$ might not be optimal for all patients 
with ASD [1]. Lafage et al. [26] demonstrated that age affects the spinopelvic alignment goals and that younger patients have a greater normal range of alignment than older patients. Selecting age-appropriate alignment goals can help prevent PJK because it can minimize the rate of overcorrection [17,37]. To avoid undercorrection or overcorrection, adjusting for age-appropriate alignment goals and avoiding overly strict adherence to PI-LL mismatch corrections could be crucial for preventing the subsequent development of PJK [5,38]. However, no clear criteria have been established for age-appropriate alignment, and methods for restoring the optimal sagittal alignment without overcorrection during the operation should be further studied [39].

The criterion of the Scoliosis Research Society-Schwab classification considers not only PT and PI/LL mismatch as linear numerical values but also the complexity of compensatory mechanisms implying the possible misleading of sagittal modifiers. To address these limitations, the Global Alignment and Proportion (GAP) score was developed and introduced as a new analytical method for predicting the occurrence of postoperative mechanical complications [40]. The GAP score is a continuum of states that provides a PI-based proportional (rather than absolute numeric) indication of pelvic version, of the magnitude and distribution of LL, and of the global spinopelvic alignment to assess disproportion compared with the calculated "ideal" for any given individual [40]. This proportion concept was expanded from age-appropriate alignment goals to personalized radiological targets reflecting the complexity of the individual human anatomy and mechanisms [41]. Therefore, understanding the GAP score is important for preventing PJK in the view of current radiological concepts.

The main characteristic of the GAP score system is that it denotes "normal" and "pathologic" standing sagittal alignment and shape as a single score for every magnitude of PI. The GAP score system assigns scores to the following factors: relative pelvic version; relative LL; lordosis distribution index; relative spinopelvic alignment; and age. A correlation has been reported between mechanical complications after surgery according to the GAP score system and associated HRQoL scores, such as the ODI, the Core Outcomes Measures Index, the Scoliosis Research Society-22 spinal deformity questionnaire, and the 36-item Short Form Health Survey [40]. The GAP scoring system is expected to be able to predict postopera- tive PJK or PJF and provide good indicators or guidelines to surgeons; however, there are still disagreements as to whether the GAP scoring system reflects mechanical failure, revisional surgery rate, and quality of life outcomes [42]. Considering this, further research on GAP scoring and the validation process are necessary, and GAP scoring should be used with caution.

\section{Surgical considerations}

The consideration for surgical risk factors includes proximal implant construction, fixation strength, implant materials, PLC preservation, and meticulous dissection at the UIV [5]. To minimize PJK and PJF, soft tissue protection, choice of a valid level and proper instrumentation at the UIV, prophylactic rib fixation, and vertebral cement augmentation are worth considering [7]. The strategies for preventing PJK should be carefully considered, on a caseby-case basis. Surgical strategies include vertebroplasty, transverse process hook fixation, terminal rod contouring, and ligament augmentation [5].

Using pedicle screws as rigid constructs increases the risk of facet violation [43]. The hybrid use of transverse hook fixation on the UIV provides a soft stress transition to the UIV and allows for less dissection of the surrounding muscle and facets [5]. In a biomechanical study of a cadaver performed by Metzger et al. [44], the use of supralaminar hooks at the top of a multilevel posterior fusion construct reduced the stress at the proximal uninstrumented motion segment $([17 \% \pm 11.5 \%]$ compared with the hybrid $[19 \% \pm 8.2 \%]$ and bilateral screw $[23 \% \pm 8.3 \%])$.

Ligament augmentation on spinous process augmentation with a polyester fiber (Mersilene tape; Ethicon, Somerville, NJ, USA) suture loop at the UIV-1, UIV, and UIV+1 level provides strength, which reduces the junctional stress at these levels [17]. Bess et al. [45] investigated the ability of posterior anchored polyethylene tethers to distribute proximal motion segment stiffness in long instrumented spine constructs in the thoracolumbar spine using a finite-element model. In their biomechanical study employing a cadaver, Kim et al. [46] stated that the posterior ligamentous tension band's role in mitigating $\mathrm{PJK}$ is secondary to the anterior column support provided by the vertebral body and intervertebral disc.

Proximal rod contouring after pedicle screw and transverse hook fixation prevents additional loading forces to the construct and minimizes the risk of junctional stress 
and screw pullout $[4,5,10]$. We recommend careful and meticulous kyphotic bending of the proximal portions of the rods such that they lay fully seated within the screw heads at the proximal two levels to prevent the preload by forcing an undercontoured rod [17]. In addition, PJK can reportedly be reduced by using a transition rod (a rod with a change in diameter or change in strength) [47]. Results from a finite-element study by Cahill et al. [47] demonstrated that the use of a transition rod at the UIV+1 level reduced the concentration of kyphosis-producing forces above the UIV that could potentially lead to PJK [48]. However, transition rods and pedicle screws fitted for these rods applicable to the clinical field have yet to be widely used, and the clinical results for these devices are scarce.

Vertebroplasty as a technique for cement augmentation at the UIV or UIV+1 levels has been reported to prevent $\mathrm{PJK}$ by reducing the risk of junctional fractures in patients with osteoporosis. Hart et al. [49] reported that vertebroplasty was associated with a low incidence of PJF. There is little guideline for surgeons in determining on how many levels to perform prophylactic cement augmentation. Considering the biomechanics of augmenting different numbers of vertebral levels and due to complications such as reduced intervertebral disc nutrition and altered loading mechanics, the authors recommend performing cement augmentation on the UIV and/or UIV+1 [50].

The advantages of cobalt chromium $(\mathrm{CoCr})$ rods include increased rod stiffness, strengthening of the stability of the spinal column construct, and preventing rod breakage [50]. However, these advantages also cause PJK due to the higher rigidity around the UIV and UIV+1 vertebral body [5,51]. Taking into account the patient's characteristics (e.g., osteoporosis, age, and BMI) and various surgical methods (e.g., osteotomy, anteroposterior fixation, and postoperative problems such as screw and rod breakage), the rod properties should be carefully selected. Stiff rods, such as those made of stainless steel or CoCr, are not always the best choice [7]. We recommend employing a titanium alloy rod considering the high rigidity of $\mathrm{CoCr}$ and stainless rods, the difficulty in proper bending, and the pulling out of screws that can occur in the process of construction. If rod reinforcement at the surgical site is required, we suggest applying a multiple rod system to reduce PJK [52].

Careful soft tissue dissection to preserve the interspinous ligaments, supraspinous ligaments, and supra- adjacent facets and their capsules at the UIV should be considered to reduce the risk of PJK and PJF [34,53]. Therefore, determining the range of exposure and minimizing soft tissue damage should be considered preoperatively [7]. Appropriate UIV selection should be carefully considered to reduce the incidence of revision surgery [57]. A UIV at T8 or lower increases the risk of PJK, and a proximal UIV could cause ASD complications [4-6]. Extended fusion to the upper thoracic level with thoracic hyperkyphosis reduces the risk of proximal junctional problems, which implies that thoracic hyperkyphosis can be regarded as a risk factor $[54,55]$.

The recent trend of employing minimally invasive surgery (MIS) for ASD is associated with minimal scarring, shorter hospital stays, and lower intraoperative blood loss [56]. Despite the powerful correction of open conventional surgery for ASD, it can also be associated with a high rate of PJK due to the more invasive surgical approach and greater implant-related factors compared with the MIS technique [57]. MIS has advantages, such as preservation of paraspinal musculatures, stripping of tendons, and denervation, which, in terms of mechanical complications, is intended to decrease approach-related thermal injury and crushing injury. Moreover, MIS procedures do not require large incisions or extensive muscular dissection, which reduces the risk of PJK [58]. Therefore, the proper choice of MIS technique by the spinal specialist during ASD surgery could maximize the benefit and minimize the risk of PJK.

\section{Conclusions}

Advances in implant technology, surgical skills, and improving surgical expertise have enabled long-level fusion and instrumentation to be implemented for ASD treatment. However, these advances in ASD surgery have also led to new complications such as proximal junctional problems, which are multifactorial in origin, stemming from a combination of surgical, radiological, and patientrelated risk factors. Based on an understanding of the modifiable factors for reducing the risk of $\mathrm{PJK}$, prevention strategies are critical for patients with ASD.

\section{Conflict of Interest}

No potential conflict of interest relevant to this article was reported. 


\section{References}

1. Diebo BG, Shah NV, Boachie-Adjei O, et al. Adult spinal deformity. Lancet 2019;394:160-72.

2. Naresh-Babu J, Viswanadha AK, Ito M, Park JB. What should an ideal adult spinal deformity classification system consist of?: review of the factors affecting outcomes of adult spinal deformity management. Asian Spine J 2019;13:694-703.

3. Pumberger M, Schmidt H, Putzier M. Spinal deformity surgery: a critical review of alignment and balance. Asian Spine J 2018;12:775-83.

4. Kim HJ, Iyer S. Proximal junctional kyphosis. J Am Acad Orthop Surg 2016;24:318-26.

5. Safaee MM, Osorio JA, Verma K, et al. Proximal junctional kyphosis prevention strategies: a video technique guide. Oper Neurosurg (Hagerstown) 2017;13:581-5.

6. Lee J, Park YS. Proximal junctional kyphosis: diagnosis, pathogenesis, and treatment. Asian Spine J 2016;10:593-600.

7. Hyun SJ, Lee BH, Park JH, Kim KJ, Jahng TA, Kim HJ. Proximal junctional kyphosis and proximal junctional failure following adult spinal deformity surgery. Korean J Spine 2017;14:126-32.

8. Cho SK, Shin JI, Kim YJ. Proximal junctional kyphosis following adult spinal deformity surgery. Eur Spine J 2014;23:2726-36.

9. Ton A, Alluri RK, Kang HP, Kim A, Hah RJ. Comparison of proximal junctional failure and functional outcomes across varying definitions of proximal junctional kyphosis. World Neurosurg 2021;146:e100-5.

10. Lau D, Clark AJ, Scheer JK, et al. Proximal junctional kyphosis and failure after spinal deformity surgery: a systematic review of the literature as a background to classification development. Spine (Phila Pa 1976) 2014;39:2093-102.

11. Glattes RC, Bridwell KH, Lenke LG, Kim YJ, Rinella A, Edwards C 2nd. Proximal junctional kyphosis in adult spinal deformity following long instrumented posterior spinal fusion: incidence, outcomes, and risk factor analysis. Spine (Phila Pa 1976) 2005;30:1643-9.

12. Helgeson MD, Shah SA, Newton PO, et al. Evaluation of proximal junctional kyphosis in adolescent idiopathic scoliosis following pedicle screw, hook, or hybrid instrumentation. Spine (Phila Pa 1976)
2010;35:177-81.

13. Hostin R, McCarthy I, O’Brien M, et al. Incidence, mode, and location of acute proximal junctional failures after surgical treatment of adult spinal deformity. Spine (Phila Pa 1976) 2013;38:1008-15.

14. O'Shaughnessy BA, Bridwell KH, Lenke LG, et al. Does a long-fusion "T3-sacrum" portend a worse outcome than a short-fusion "T10-sacrum" in primary surgery for adult scoliosis? Spine (Phila Pa 1976) 2012;37:884-90.

15. Bridwell KH, Lenke LG, Cho SK, et al. Proximal junctional kyphosis in primary adult deformity surgery: evaluation of 20 degrees as a critical angle. Neurosurgery 2013;72:899-906.

16. Hart R, McCarthy I, O'Brien M, et al. Identification of decision criteria for revision surgery among patients with proximal junctional failure after surgical treatment of spinal deformity. Spine (Phila Pa 1976) 2013;38:E1223-7.

17. Nguyen NL, Kong CY, Hart RA. Proximal junctional kyphosis and failure: diagnosis, prevention, and treatment. Curr Rev Musculoskelet Med 2016;9:299308.

18. Yagi M, Rahm M, Gaines R, et al. Characterization and surgical outcomes of proximal junctional failure in surgically treated patients with adult spinal deformity. Spine (Phila Pa 1976) 2014;39:E607-14.

19. Kim YJ, Bridwell KH, Lenke LG, Glattes CR, Rhim S, Cheh G. Proximal junctional kyphosis in adult spinal deformity after segmental posterior spinal instrumentation and fusion: minimum five-year follow-up. Spine (Phila Pa 1976) 2008;33:2179-84.

20. Kim JS, Phan K, Cheung ZB, et al. Surgical, radiographic, and patient-related risk factors for proximal junctional kyphosis: a meta-analysis. Global Spine J 2019;9:32-40.

21. Pennington Z, Cottrill E, Ahmed AK, et al. Paraspinal muscle size as an independent risk factor for proximal junctional kyphosis in patients undergoing thoracolumbar fusion. J Neurosurg Spine 2019;31:380-8.

22. Kim HJ, Yang JH, Chang DG, et al. Adult spinal deformity: current concepts and decision-making strategies for management. Asian Spine J 2020;14:886-97.

23. Barrey C, Roussouly P, Perrin G, Le Huec JC. Sagittal balance disorders in severe degenerative spine: can we identify the compensatory mechanisms? Eur 
Spine J 2011;20(Suppl 5):626-33.

24. Roussouly P, Gollogly S, Berthonnaud E, Dimnet J. Classification of the normal variation in the sagittal alignment of the human lumbar spine and pelvis in the standing position. Spine (Phila Pa 1976) 2005;30:346-53.

25. Sebaaly A, Gehrchen M, Silvestre C, et al. Mechanical complications in adult spinal deformity and the effect of restoring the spinal shapes according to the Roussouly classification: a multicentric study. Eur Spine J 2020;29:904-13.

26. Lafage R, Schwab F, Glassman S, et al. Age-adjusted alignment goals have the potential to reduce PJK. Spine (Phila Pa 1976) 2017;42:1275-82.

27. Kim HJ, Yagi M, Nyugen J, Cunningham ME, Boachie-Adjei O. Combined anterior-posterior surgery is the most important risk factor for developing proximal junctional kyphosis in idiopathic scoliosis. Clin Orthop Relat Res 2012;470:1633-9.

28. Maruo K, Ha Y, Inoue S, et al. Predictive factors for proximal junctional kyphosis in long fusions to the sacrum in adult spinal deformity. Spine (Phila Pa 1976) 2013;38:E1469-76.

29. Aydogan M, Ozturk C, Karatoprak O, Tezer M, Aksu $\mathrm{N}$, Hamzaoglu A. The pedicle screw fixation with vertebroplasty augmentation in the surgical treatment of the severe osteoporotic spines. J Spinal Disord Tech 2009;22:444-7.

30. Tan JS, Kwon BK, Dvorak MF, Fisher CG, Oxland TR. Pedicle screw motion in the osteoporotic spine after augmentation with laminar hooks, sublaminar wires, or calcium phosphate cement: a comparative analysis. Spine (Phila Pa 1976) 2004;29:1723-30.

31. Kim DK, Kim JY, Kim DY, Rhim SC, Yoon SH. Risk factors of proximal junctional kyphosis after multilevel fusion surgery: more than 2 years follow-up data. J Korean Neurosurg Soc 2017;60:174-80.

32. Anderson AL, McIff TE, Asher MA, Burton DC, Glattes RC. The effect of posterior thoracic spine anatomical structures on motion segment flexion stiffness. Spine (Phila Pa 1976) 2009;34:441-6.

33. Cammarata M, Aubin CE, Wang X, Mac-Thiong JM. Biomechanical risk factors for proximal junctional kyphosis: a detailed numerical analysis of surgical instrumentation variables. Spine (Phila Pa 1976) 2014;39:E500-7.

34. Glassman SD, Bridwell K, Dimar JR, Horton W, Ber- ven S, Schwab F. The impact of positive sagittal balance in adult spinal deformity. Spine (Phila Pa 1976) 2005;30:2024-9.

35. Schwab F, Ungar B, Blondel B, et al. Scoliosis Research Society-Schwab adult spinal deformity classification: a validation study. Spine (Phila Pa 1976) 2012;37:1077-82.

36. Schwab F, Lafage V, Patel A, Farcy JP. Sagittal plane considerations and the pelvis in the adult patient. Spine (Phila Pa 1976) 2009;34:1828-33.

37. Iwamae M, Matsumura A, Namikawa T, et al. Surgical outcomes of multilevel posterior lumbar interbody fusion versus lateral lumbar interbody fusion for the correction of adult spinal deformity: a comparative clinical study. Asian Spine J 2020;14:421-9.

38. Kim WJ, Lee JW, Park KY, Chang SH, Song DG, Choy WS. Treatment of adult spinal deformity with sagittal imbalance using oblique lumbar interbody fusion: can we predict how much lordosis correction is possible? Asian Spine J 2019;13:1017-27.

39. Kim HJ, Bridwell KH, Lenke LG, et al. Patients with proximal junctional kyphosis requiring revision surgery have higher postoperative lumbar lordosis and larger sagittal balance corrections. Spine (Phila Pa 1976) 2014;39:E576-80.

40. Yilgor C, Sogunmez N, Boissiere L, et al. Global Alignment and Proportion (GAP) score: development and validation of a new method of analyzing spinopelvic alignment to predict mechanical complications after adult spinal deformity surgery. J Bone Joint Surg Am 2017;99:1661-72.

41. Jacobs E, van Royen BJ, van Kuijk SM, et al. Prediction of mechanical complications in adult spinal deformity surgery-the GAP score versus the Schwab classification. Spine J 2019;19:781-8.

42. Kwan KY, Lenke LG, Shaffrey CI, et al. Are higher global alignment and proportion scores associated with increased risks of mechanical complications after adult spinal deformity surgery?: an external validation. Clin Orthop Relat Res 2021;479:312-20.

43. Hyun SJ, Rhim SC. Clinical outcomes and complications after pedicle subtraction osteotomy for fixed sagittal imbalance patients: a long-term follow-up data. J Korean Neurosurg Soc 2010;47:95-101.

44. Metzger MF, Robinson ST, Svet MT, Liu JC, Acosta FL. Biomechanical analysis of the proximal adjacent segment after multilevel instrumentation of the 
thoracic spine: do hooks ease the transition? Global Spine J 2016;6:335-43.

45. Bess S, Harris JE, Turner AW, et al. The effect of posterior polyester tethers on the biomechanics of proximal junctional kyphosis: a finite element analysis. J Neurosurg Spine 2017;26:125-33.

46. Kim JS, Cheung ZB, Arvind V, Caridi J, Cho SK. Role of posterior ligamentous reinforcement in proximal junctional kyphosis: a cadaveric biomechanical study. Asian Spine J 2019;13:68-76.

47. Cahill PJ, Wang W, Asghar J, et al. The use of a transition rod may prevent proximal junctional kyphosis in the thoracic spine after scoliosis surgery: a finite element analysis. Spine (Phila Pa 1976) 2012;37:E68795.

48. Nguyen TQ, Buckley JM, Ames C, Deviren V. The fatigue life of contoured cobalt chrome posterior spinal fusion rods. Proc Inst Mech Eng H 2011;225:194-8.

49. Hart RA, McCarthy I, Ames CP, Shaffrey CI, Hamilton DK, Hostin R. Proximal junctional kyphosis and proximal junctional failure. Neurosurg Clin N Am 2013;24:213-8.

50. Han S, Hyun SJ, Kim KJ, Jahng TA, Kim HJ. Comparative study between cobalt chrome and titanium alloy rods for multilevel spinal fusion: proximal junctional kyphosis more frequently occurred in patients having cobalt chrome rods. World Neurosurg 2017;103:4049.

51. Kayanja MM, Schlenk R, Togawa D, Ferrara L, Lieberman I. The biomechanics of 1, 2, and 3 levels of vertebral augmentation with polymethylmethacrylate in multilevel spinal segments. Spine (Phila Pa 1976) 2006;31:769-74.
52. Han S, Hyun SJ, Kim KJ, Jahng TA, Lee S, Rhim SC. Rod stiffness as a risk factor of proximal junctional kyphosis after adult spinal deformity surgery: comparative study between cobalt chrome multiple-rod constructs and titanium alloy two-rod constructs. Spine J 2017;17:962-8.

53. Kim WJ, Lee JW, Kim SM, et al. Precautions for combined anterior and posterior long-level fusion for adult spinal deformity: perioperative surgical complications related to the anterior procedure (oblique lumbar interbody fusion). Asian Spine J 2019;13:82331.

54. Ha Y, Maruo K, Racine L, et al. Proximal junctional kyphosis and clinical outcomes in adult spinal deformity surgery with fusion from the thoracic spine to the sacrum: a comparison of proximal and distal upper instrumented vertebrae. J Neurosurg Spine 2013;19:360-9.

55. Lee JH, Kim JU, Jang JS, Lee SH. Analysis of the incidence and risk factors for the progression of proximal junctional kyphosis following surgical treatment for lumbar degenerative kyphosis: minimum 2-year follow-up. Br J Neurosurg 2014;28:252-8.

56. Yang JH, Chang DG, Suh SW, et al. Safety and effectiveness of minimally invasive scoliosis surgery for adolescent idiopathic scoliosis: a retrospective case series of 84 patients. Eur Spine J 2020;29:761-9.

57. Yen CP, Mosley YI, Uribe JS. Role of minimally invasive surgery for adult spinal deformity in preventing complications. Curr Rev Musculoskelet Med 2016;9:309-15.

58. Mummaneni PV, Park P, Fu KM, et al. Does minimally invasive percutaneous posterior instrumentation reduce risk of proximal junctional kyphosis in adult spinal deformity surgery?: a propensity-matched cohort analysis. Neurosurgery 2016;78:101-8. 